\title{
Mining Association Rules in Multiple Relations
}

\author{
Luc Dehaspe and Luc De Raedt \\ Katholieke Universiteit Leuven, Department of Computer Science, \\ Celestijnenlaan 200A, B-3001 Heverlee, Belgium \\ email : \{Luc.Dehaspe, Luc.DeRaedt\}@cs.kuleuven.ac.be \\ fax : ++3216327996 ; telephone : ++3216327567
}

\begin{abstract}
The application of algorithms for efficiently generating association rules is so far restricted to cases where information is put together in a single relation. We describe how this restriction can be overcome through the combination of the available algorithms with standard techniques from the field of inductive logic programming. We present the system WARMR, which extends APRIORI [2] to mine association rules in multiple relations. We apply WARMR to the natural language processing task of mining part-of-speech tagging rules in a large corpus of English.
\end{abstract}

Keywords: association rules, inductive logic programming

\section{Introduction}

Association rules are generally recognized as a highly valuable type of regularities and various algorithms have been presented for efficiently mining them in large databases (cf. $[1,7,2])$. To the best of our knowledge, the application of these algorithms is so far restricted to cases where information is put together in a single relation. We describe how this restriction can be overcome through the combination of the available algorithms with standard techniques from the field of inductive logic programming $[6,3,8]$.

In Section 2, we first formalize the concept of association rules over multiple relations and the task of mining them in a deductive relational database. In Section 3, we present the algorithm WARMR, which extends APRIORI [2] to mine association rules in multiple relations. WARMR is applied in Section 4 to a database of Wall Street Journal articles.

\section{Representation and Problem specification}

We first recapitulate some original concepts of association rules over a single relation, and then generalize these to the case of association rules over multiple relations.

\section{$2.1 \quad$ Association Rules}

Agrawal, Imielinski, and Swami [1] define association rules as expressions of the form $X \Rightarrow Y$, where $X$ and $Y$ are itemsets, i.e. sets of attributes. Association 
rules are evaluated with respect to a relation $\mathcal{R}$ over a set $\mathcal{I}$ of binary attributes. Each example in $\mathcal{R}$ is associated with a unique key, below called the ExampleKey $E x K e y$, and with an itemset $E \subseteq \mathcal{I}$ that contains all attributes with value 1 . A rule $X \Rightarrow Y$ over relation $\mathcal{R}$ then holds with confidence $c$ if $c \%$ of the itemsets in $\mathcal{R}$ that are supersets of $X$ are also supersets of $Y$. The support of this rule equals $s$, if $s \%$ of the examples in $\mathcal{R}$ are supersets of $X \cup Y$.

For instance, given the relation kid(KID, Piglet, Dolphin, Icecream), the rule piglet, icecream $\Rightarrow$ dolphin (c:85,s:9) states that $85 \%$ of the kid-examples that like Piglet and icecream, also like dolphins, and that $9 \%$ of the kid-examples are fond of Piglet, icecream and dolphins.

The task of discovering association rules in a single relation $\mathcal{R}$ is to generate all association rules over $\mathcal{R}$ with confidence and support above given thresholds, below called minconf and minsup respectively. Efficient algorithms for solving this task proceed in two stages (cf. [1]). First, all large itemsets are generated, i.e. all itemsets with support above minsup. Second, the itemsets found large in the first stage are used to generate the target association rules without further consideration of the data in $\mathcal{R}$. This second step is based on the observation that $X \Rightarrow Y$ holds if and only if $X$ and $X \cup Y$ are amongst the large itemsets, and $\operatorname{support}(X \cup Y) / \operatorname{support}(Y) \geq \operatorname{minconf}$.

\subsection{Association Rules over Multiple Relations}

Let us now modify the original concepts, so as to make them operational in the context of a deductive relational database $\mathcal{D}$ composed of multiple relations.

As before, each example in $\mathcal{D}$ is identified by a unique ExampleKey ExKey. Furthermore, all relations in $\mathcal{D}$ have exactly one attribute that corresponds to $E x K e y$. The idea is that the ExKey then partitions the tuples in a deductive relational database into disjoint subsets with identical ExKey. An example identified by ExampleKey $i$ is then the set of all tuples in the database with $E x K e y=i$. Notice the ExampleKey is somewhat similar to notion of a clustering field as used in the database literature. The idea of partitioning the database also fits in the learning from interpretations paradigm (an interpretation here corresponds to a partition), introduced by [5] and related to other inductive logic programming settings in [4]. From a practical point of view a significant speed up can be obtained if the partioning scheme creates examples that fit in main memory. As we will see later, the algorithm for efficiently mining association rules over multiple relations exploits and crucially depends on the fact that an example can be singled out from the database.

Association rules over multiple relations (ARMR's) are expressions of the form $X \Rightarrow Y$, where $X$ and $Y$ are atomsets, i.e. sets of logical atoms of the form $p\left(t_{1}, \ldots, t_{n}\right)$, where each term $t_{i}$ is a variable $V$ or a function $f\left(t_{1}, \ldots, t_{k}\right)$ (if $\mathrm{k}=0, \mathrm{f}$ is a constant). Given an atomset $A=\left\{A_{1}, \ldots, A_{m}\right\}$, and an example $E_{i}$ with $E x K e y=i$ we will associate with the couple $\left(A, E_{i}\right)$ an existentially quantified conjunction (eqc) of the form

$$
e q c\left(A, E_{i}\right)=\exists\left(E x K e y=i \wedge A_{1} \wedge \ldots \wedge A_{m}\right) .
$$




\begin{tabular}{|c|c|c|c|c|c|c|}
\hline & ES & & HAS & \multicolumn{3}{|c|}{ PREFERS } \\
\hline KID & OBJECT & KID & OBJECT & KID & OBJECT & $\overline{\mathrm{TO}}$ \\
\hline joni & ice & joni & & joni & & puddin \\
\hline joni & dolph & joni & pig & joni & ing & raisins \\
\hline elliot & piglet & elliot & icecream & jo & gir & gnu \\
\hline int & 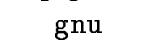 & & & ellio & li & icecream \\
\hline elliot & lion & & & elliot & piglet & dolphin \\
\hline
\end{tabular}

Fig. 1. A sample from the KIDS database

We say that an atomset $A$ covers an example $E_{i}$ if eqc $\left(A, E_{i}\right)$ is true in $\mathcal{D}$. Several strategies are available to verify truth of $e q c\left(A, E_{i}\right)$ in $\mathcal{D}$. One possibility is to convert eqc $\left(A, E_{i}\right)$ into an SQL-query. In our implementation, we have opted for a conversion of eqc $\left(A, E_{i}\right)$ to a Prolog query $\leftarrow e q c\left(A, E_{i}\right)$. If this Prolog query succeeds in $\mathcal{D}$, the atomset $A$ covers example $E_{i}$, if the query fails ${ }^{1}$, the atomset does not cover example $E_{i}$.

An ARMR $X \Rightarrow Y$ then holds in $\mathcal{D}$ with confidence $c$ if $c \%$ of the examples in $\mathcal{D}$ that are covered by $X$ are also covered by $X \cup Y^{2}$. The support of this ARMR equals $s$, if $s \%$ of the examples in $\mathcal{D}$ are covered by $X \cup Y$.

Consider for instance the KIDS database in Figure 1. Let the ExampleKey be KID. Notice the relations likes(KID, Object), has(KID, Object), and prefers (KID, Object, Above) each have multiple entries per ExampleKey.

As shown in Figure 2, the ExampleKey KID, indeed partitions the KIDS database in disjoint examples for ExKey = joni and ExKey=elliot. With this representation, the running example rule would take the form

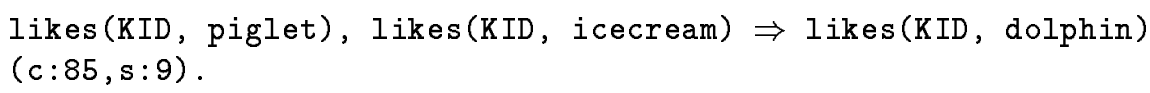

A new rule over all three relations might be

$$
\text { likes(KID, A), has (KID, B) } \Rightarrow \text { prefers (KID, A, B) (c:98, s:70). }
$$

This new rule states that $98 \%$ of the kids who like something and have something prefer a thing they like over a thing they have. Furthermore, $70 \%$ of the kids like and have something and prefer the first over the second.

The task of discovering ARMR's in a deductive relational database $\mathcal{D}$ is then to generate all ARMR's over $\mathcal{D}$ with confidence above minconf and support above minsup. In the next section we show how the two-phased algorithm of [1] summarized above can be modified for solving this task.

\footnotetext{
${ }^{1}$ Termination can be guaranteed by employing a depth-bound on the depth of the proof tree.

${ }^{2}$ Notice we evaluate $X \cup Y$, rather than just $Y$, to allow variables occurring in $Y$ to be bound in $X$.
} 


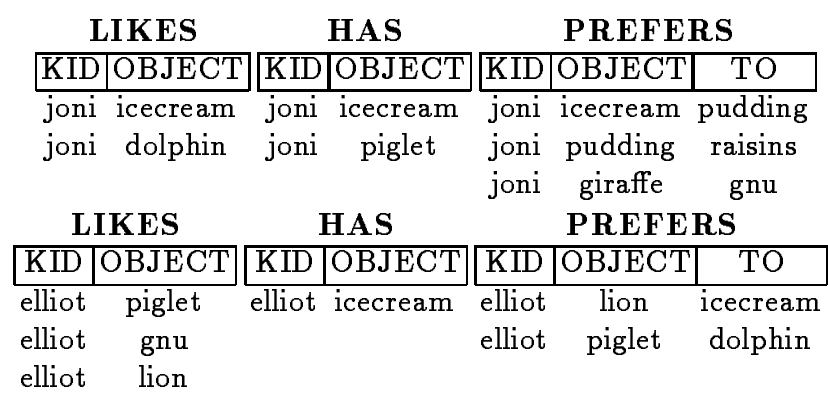

Fig. 2. Two disjoint examples from the KIDS database.

\section{Mining Large Atomsets: Algorithm WARMR}

In this section we demonstrate how an existing algorithm for mining large itemsets, i.e. itemsets with support above minsup, can be modified to mine large atomsets over multiple relations. As a starting point we take the APRIORI algorithm by Agrawal, Mannila, Srikant, Toivonen, and Verkamo [2]. We will stick as closely as possible to the notation introduced by these authors.

Essentially APRIORI (WARMR) exploits the lattice structure the subset relation imposes on the space of itemsets (atomsets ${ }^{3}$ ) to perform a breadth-first search. The symbol $\mathcal{L}_{k}$ is used to refer to the set of large $k$-itemsets mined at depth $k$ in this lattice. Given $\mathcal{L}_{k-1}$ the next search level $k$ is prepared with the generation of all potentially large candidates $\mathcal{C}_{k}$. The general idea here is to prune the set of $k$-itemsets, from what we know of the large $(k-1)$-itemsets. Initial candidate set $\mathcal{C}_{1}$ is to be specified by the user. The actual support of all sets in $\mathcal{C}_{k}$ is then calculated during a single pass over the data. From $\mathcal{C}_{k}$ we can select the sets with minimum support into $\mathcal{L}_{k}$, and move on to the next level.

The main loop of WARMR is formalized in Algorithm 1. The original algorithm APRIORI has been modified in two places. First, at step (3.a) an explicit reference is added to the fact that an example $E$ is singled out from the database. This step is trivial in APRIORI where an example is simply a tuple. As described in Section 2.2, when mining ARMR's this step involves selecting from all relations the tuples with a given value for the ExampleKey. In our implementation the main loop is executed in Prolog and an example is loaded in Prolog (and main memory) from an Oracle $7^{T M}$ database. Alternative implementations should somehow succeed in isolating (activating) a single example, such that rules are evaluated with respect to the (small) example instead of the (large) database.

Step (3.b) is the second location where WARMR deviates from the original: a coverage test involving theorem proving replaces the original subset check. An important observation here is that in case atoms share variables apart from

\footnotetext{
${ }^{3}$ In the case of atomsets the subset relation can be interpreted as a special case of the $\theta$-subsumption relation [10] frequently used in inductive logic programming.
} 
Algorithm 1 : WARMR

Inputs: Set of candidate 1-atomsets $\mathcal{C}_{1}$; database $\mathcal{D}$; minsup

Outputs: All queries with minimum support $\bigcup_{k} \mathcal{L}_{k}$

1. Initialize $k:=1$

2. For each candidate $C_{i} \in \mathcal{C}_{k}$, initialize support counter sup $_{i}:=0$

3. For each example $E \in \mathcal{D}$, do the following:

(a) load $E$

(b) For each candidate $k$-atomset $C_{i} \in \mathcal{C}_{k}$, do the following:

If atomset $C_{i}$ covers $E$, increment support counter sup $_{i}$

4. Let set of large $k$-atomsets $\mathcal{L}_{k}:=\left\{\left(C_{i}\right.\right.$, sup $\left._{i}\right) \in \mathcal{C}_{k} \times[0,100] \mid$ sup $_{i} \geq$ minsup $\}$

5. Compute new candidates $\mathcal{C}_{k+1}$ from $\mathcal{L}_{k}$ using WARMR-gen

6. If $\mathcal{C}_{k+1}$ is not empty, increment $k$ and go to step 2

the ExampleKey, the coverage test should be executed on the full atomset, not on single atoms. As a consequence, the use of (an adaptation of) algorithm APRIORITID [2] (which passes through the data only once), is restricted to the case where the only shared variable is the ExampleKey.

Algorithm 2 : WARMR-gen

Inputs: Set of large $k$-atomsets $\mathcal{L}_{k}$; clausal theory $\mathcal{T}$

Outputs: Set of candidate (potentially large) $(k+1)$-atomsets $\mathcal{C}_{k+1}$

1. $\mathcal{C}_{k+1}:=\left\{\left\{p_{1}, \ldots, p_{k}, q_{k}\right\} \mid\left(\left\{p_{1}, p_{2}, \ldots, p_{k-1}, p_{k}\right\}, s_{1}\right) \in \mathcal{L}_{k}\right.$

$\left(\left\{p_{1}, p_{2}, \ldots, p_{k-1}, q_{k}\right\}, s_{2}\right) \in \mathcal{L}_{k}$

$\left.p_{k}<q_{k}\right\}$

2. Delete all $(k+1)$-atomsets $C=\left\{p_{1}, \ldots, p_{k}, q_{k}\right\}$ from $\mathcal{C}_{k+1}$ with:

(a) some $k$-subset of $C$ is not in $\mathcal{L}_{k}$; or

(b) $\mathcal{T} \wedge \exists\left(p_{1} \wedge p_{2} \wedge \ldots \wedge p_{k} \wedge q_{k}\right) \models \square(C$ is contradictory); or

(c) $\mathcal{T} \wedge \exists\left(p_{1} \wedge p_{2} \wedge \ldots \wedge p_{k} \wedge \neg q_{k}\right) \mid=\square(C$ is redundant $)$

The procedure WARMR-gen for the generation of all potentially large candidates $\mathcal{C}_{k}$ is presented in Algorithm 2. This procedure consists of a join-step (1) followed by a prune-step (2). In the join-step we assume that atomsets are kept sorted in their lexicographic order. The condition $p_{k}<q_{k}$ is added to preserve this property. From this initial set $\mathcal{C}_{k+1}$, we then, following APRIORI-gen [2], prune the atomsets that have a subset with no minimum support. New with respect to APRIORI-gen is that we have added two pruning conditions. These extra tests involve running a theorem prover to verify whether the atomsets are contradictory (2.b) or redundant (2.c) with respect to a user-specified clausal theory $\mathcal{T}$. Clausal theory $\mathcal{T}$ provides the user with a powerful tool to specify taxonomies, mutual exclusion relations, and other types of information which is 
WSJ_CORPUS

\begin{tabular}{ccccc}
\hline SID & WID & WORD & TAG \\
\hline 77 & 1 & champagne & $\mathrm{nn}$ \\
77 & 2 & and & $\mathrm{cc}$ \\
77 & 3 & dessert & $\mathrm{nn}$ \\
77 & 4 & followed & vbd \\
77 & 5 &. & .
\end{tabular}

Fig. 3. A sample from the WSJ database

known to hold in the database. Pruning steps (2.b) and (2.c) may thus contribute not only to the efficiency of the algorithm, but also to the quality of the output (cf. the novelty requirement frequently mentioned in definitions of KDD).

For the generation of ARMR's we refer to the algorithm described in [2]. The procedure ap-genrules described there for large itemsets, with minor modifications, applies to large atomsets.

\section{Application: Part-of-Speech tagging}

To apply WARMR, we established a connection between an Oracle $7^{T M}$ database and ProLog by BIM on a SPARCstation 20, to obtain the full power of a deductive relational database. We performed two experiments on the tagged Wall Street Journal (WSJ) corpus provided by the Penn Treebank Project [9]. In this corpus of Wall Street Journal articles, each of the (close to 3 million) words is associated with a so-called part-of-speech tag, chosen from set of about 50 tags. The corpus is frequently used for inductive experiments typically aimed at the (semi-) automatic construction of part-of-speech taggers.

We stored the raw WSJ data in a single-relation Oracle $7^{T M}$ database as shown in Figure 3. SID refers to the sequence number of the sentence in the corpus, and WID to the sequence number of the word in the sentence. The TAG column shows the part-of-speech assigned to WORD in that sentence. The main problem of automated tagging is that words may get different tags in different contexts (e.g. the word "plans" can be a noun or a verb). Therefore it is useful to mine the corpus for rules that might help to resolve ambiguity of a word given its context.

\subsection{Discovering properties of sentences}

In a first experiment, we let SID be the ExampleKey, such that we obtained about 125000 examples (= sentences), for the full WSJ database ( $\approx 3$ million words). The set of candidate atomsets was initialized to $C_{1}=\{\{\operatorname{determiner}(\mathrm{SID}, \mathrm{A}, \mathrm{B})\}$, $\{$ adjective (SID, C , D) $\},\{$ adjective(SID $, B, D)\},\{$ noun $(S I D, E, F)\}$, $\{$ noun (SID, D,F) $\},\{$ noun (SID, B, F ) $\}$. The relations in $C_{1}$ are defined intensionally in a straightforward manner. The second argument each time corresponds to WID in WSJ_CORPUS, and the third argument is simply the second 
argument plus 1 . The name of the relation refers to the fact a number of tags are grouped. For instance, a tuple noun(SID, X, (X+1)) exists for each tuple wsj_corpus (SID, X, $W, \mathrm{Tag}$ ) with Tag $\in\{\mathrm{nn}, \mathrm{nns}, \mathrm{prp}$, wp, nnp, nnps $\}$. In about $48 \mathrm{~h}$, WARMR found all atomsets with support above $10 \%$, on the basis of which we could generate ARMR's such as:

$$
\begin{aligned}
& \text { determiner(SID , A, B), adjective(SID , B, D) } \Rightarrow \text { noun(SID , D F ) } \\
& (\mathrm{c}: 81, \mathrm{~s}: 32 \text { ) }
\end{aligned}
$$

In words, this rule says that in $81 \%$ of the sentences in which a determiner occurs followed by an adjective, there is also a sequence determiner, adjective, noun. Furthermore, the latter sequence occurs in $32 \%$ of the sentences.

\subsection{Inducing frequent binary features}

All work on induction of part-of-speech taggers is based on the assumption that the context of target word is a good indicator for the target tag. In its most basic form, the context is simply the words in the sentence that precede and follow the target. As combinations of more than two words are typically very rare, even in huge corpora, it is common practise to generalize over word sequences and consider the more frequent (disambiguated) tag sequences (typically to the left of the target). We take this idea one step further and consider sequences of constituents, where constituents (e.g. noun phrase) generalize over tag sequences (e.g. determiner +0 or more adjectives + noun). Constituents are of variable length (unlike tags, which all have length 1), such that we need a first order language for representing sequences of constituents.

In a second experiment we deployed WARMR to the discovery of frequent sequences of constituents. These can later be used as binary features (a constituent sequence precedes the target or not) in standard propositional approaches. We used an elementary phrase structure grammar for building constituents over tag sequences. The combination SID, WID served as ExampleKey, and we retained only the first 100000 examples (= words = starting points for a constituent sequence). The support threshold was lowered to $0.5 \%$. In about 5 days WARMR discovered all 783 constituent sequences that occur at least 500 times in a 100000

\begin{tabular}{|c|c|}
\hline constituent sequence & frequency \\
\hline$\overline{\mathrm{np}(W I D, B)}$ & 36310 \\
\hline$n p(W I D, B), \operatorname{vp}(B, C)$ & 7842 \\
\hline$n p(W I D, B), \operatorname{vp}(B, C), n p(C, D)$ & 2165 \\
\hline$n p(W I D, B), \operatorname{vp}(B, C), n p(C, D), \operatorname{prep}(D, E)$ & 995 \\
\hline$n p(W I D, B), \operatorname{vp}(B, C), \operatorname{np}(C, D), \operatorname{prep}(D, E)$ & 644 \\
\hline
\end{tabular}
word corpus, such as ${ }^{4}$ :

For instance, the last line says that 644 words in the corpus are starting points for the sequence noun phrase + verb phrase + noun phrase + preposition + noun phrase.

\footnotetext{
${ }^{4}$ To simplify matters, the ExampleKey has been left out in the presentation of the sequences.
} 


\section{Concluding remarks}

We have introduced association rules over multiple relations (ARMR's) as a novel class of regularities, and presented and applied an algorithm WARMR for mining these regularities in deductive relational databases. Characteristic for WARMR is that examples are disjoint subsets of the database. The efficiency of WARMR derives from, and crucially depends on the possibility to cache a single example in main memory.

\section{Acknowledgements}

This research is part of the ESPRIT IV project no. 20237 on Inductive Logic Programming II (ILP ${ }^{2}$ ), and FWO project no. 8.0157.97 (LingUADUCT). Luc De Raedt is supported by the Fund for Scientific Research, Flanders. The authors would like to thank Walter Daelemans and Arno Knobbe for inspiring discussions on respectively part-of-speech tagging and mining association rules.

\section{References}

1. R. Agrawal, T. Imielinski, and A. Swami. Mining assosiation rules between sets of items in large databases. In Proceedings of the 1993 International Conference on Management of Data (SIGMOD 93), pages 207-216, May 1993.

2. R. Agrawal, H. Mannila, R. Srikant, H. Toivonen, and A.I. Verkamo. Fast discovery of association rules. In U. Fayyad, G. Piatetsky-Shapiro, P. Smyth, and R. Uthurusamy, editors, Advances in Knowledge Discovery and Data Mining, pages 307-328. The MIT Press, 1996.

3. L. De Raedt, editor. Advances in Inductive Logic Programming, volume 32 of Frontiers in Artificial Intelligence and Applications. IOS Press, 1996.

4. L. De Raedt. Induction in logic. In R.S. Michalski and Wnek J., editors, Proceedings of the 3rd International Workshop on Multistrategy Learning, pages 29-38, 1996.

5. L. De Raedt and S. Džeroski. First order $j k$-clausal theories are PAC-learnable. Artificial Intelligence, 70:375-392, 1994.

6. S. Džeroski. Inductive logic programming and knowledge discovery in databases. In U. Fayyad, G. Piatetsky-Shapiro, P. Smyth, and R. Uthurusamy, editors, $A d$ vances in Knowledge Discovery and Data Mining, pages 118-152. The MIT Press, 1996.

7. M. Houtsma and A. Swami. Set-oriented mining of assocation rules. Technical Report RJ 9567, IBM Almaden Research Center, San Jose, Calif., 1993.

8. N. Lavrač and S. Džeroski. Inductive Logic Programming: Techniques and Applications. Ellis Horwood, 1994.

9. M.P. Marcus, B. Santorini, and M. A. Marcinkiewicz. Building a large annotated corpus of English: the Penn Treebank. Computational Linguistics, 19(2):313-330, 1993.

10. G. Plotkin. A note on inductive generalization. In Machine Intelligence, volume 5, pages 153-163. Edinburgh University Press, 1970.

This article was processed using the $\mathrm{I}_{\mathrm{H}}^{\mathrm{T}} \mathrm{T} \mathrm{X}$ macro package with LLNCS style 